\title{
ALGORITHM EXAMPLES IN FINANCIAL MARKETS: ASSETS INCOME ANALYZING, BENCHMARK AND BACKTESTING WITH PYTHON PROGRAMMING
}

\author{
DOI: 10.17261/Pressacademia.2020.1364 \\ PAP- V.12-2020(28)-p.95-97
}

\section{Selahaddin Bilal Ozgur ${ }^{1}$, Ayben Koy ${ }^{2}$}

${ }^{1}$ Istanbul Commerce University, Finance Institute, Department of Capital Markets, Sutluce Campus, Istanbul, Turkey. sebiozgur@windowslive.com, ORCID: 0000-0003-0924-4792

${ }^{2}$ Istanbul Commerce University, Faculty of Business, Banking and Finance Program, Sutluce Campus, Istanbul, Turkey. akoy@ticaret.edu.tr, ORCID: 0000-0002-2506-6634

To cite this document

Ozgur, S.B., Koy, A.,, (2020). Algorithm examples in financial markets: assets income analysing, benchmark and acktesting with python programmimg. PressAcademia Procedia (PAP), V.12, p.95-97.

Permanent link to this document: http://doi.org/10.17261/Pressacademia.2020.1364

Copyright: Published by PressAcademia and limited licensed re-use rights only.

\section{ABSTRACT}

Purpose- The need for fast and reliable trading has increased to reduce human and non-human risks in financial markets and to change the pricing in the markets. Algorithms are prepared and used as a result of technological opportunities to manage these risks and to facilitate the management of revenue policies. Strategies can be created based on historical information on asset prices and these strategies can be implemented through algorithms. This study aims to analyze the performance of the applications of the created strategies with algorithms. Methodology- Based on the prices that occurred in the market within a certain period, instead of "keep in portfolio", the results of the buysell transactions made in accordance with the condition-condition principle such as golden cross of algorithmic trade transactions were compared. The program, in which 50-day and 200-day moving averages are used in algorithmic transactions, was written in Python (vs. 2.7). Findings- By using the same, similar or different algorithms for different asset types, different rates of positive and negative returns can be obtained.The results obtained provide evidence that algorithmic transactions can perform better against the acquisition and retention of the asset in the portfolio.

Conclusion- In the study comparing algorithmic transactions with the "keep in portfolio" investment strategy, varying results were obtained for different assets. Although the findings show that the same strategy cannot bring positive returns for every financial instrument, it is recommended to develop strategies specific to different asset groups for future studies.

Keywords: Algorithmic trade, benchmark and backtest, assets income analyzing, data science

JEL Codes: D53, F47, G11,

\section{FINANSAL PIYASALARDA ALGORITMA UYGULAMALARI: PYTHON PROGRAMLAMA ILE GETiRi ANALIZI, KIYASLAMA VE KARŞILAŞTIRMA}

\section{ÖZET}

Amaç- Finansal piyasalarda beşeri ve beşeri olmayan risklerin azaltılması ve piyasalardaki fiyatlamaların değişimlerine yönelik hızlı ve güvenilir bir şekilde alım-satım yapma ihtiyacı artmıştır. Söz konusu risklerin yönetilmesi ve getiri politikalarının yönetiminin kolaylaştırılması için teknolojik imkanlar neticesinde algoritmalar hazırlanmakta ve kullanılmaktadır. Varlık fiyatlarındaki geçmiş bilgiye göre stratejiler oluşturulabilmekte ve bu stratejiler algoritmalar vasıtasıyla uygulanabilmektedir. Bu çalışmanın amacı, oluşturulan stratejilerin algoritmalar vasıtasıyla yapılan uygulamalarının performanslarını analiz etmektir.

Yöntem- Belirli bir dönem içerisinde piyasada oluşan fiyatlamalar baz alınarak "portföyde tut" yerine algoritmik trade işlemlerinin golden cross vb. Koşul-şart ilkesi doğrultusunda yapılan al-sat işlemleri sonuçları mukayese edilmiştir. Algoritmik işlemlerde 50 günlük ve 200 günlük hareketli ortalamaların kullanıldığı program, Python dilinde (vs. 2.7) yazılmıştır.

Bulgular- Farklı varlık türlerinde aynı, benzer veya farklı algoritmalar kullanılarak farklı oranlarda pozitif veya negative getiriler elde edilebilmektedir. Elde edilen sonuçlar, algoritmik işlemlerin varlığın portföye alınıp tutulmasına karşı daha yüksek performans gösterebileceğine dair kanıtlar sunmaktadır.

Sonuç- Algoritmik işlemlerin "portföyde tut” yatırım stratejisi ile karşılaştırıldığı çalışmada, farklı varlıklar için değişen sonuçlar elde edilmiştir. Bulgular, her finansal enstrüman için aynı stratejinin pozitif getiriye ulaştıramaycağını göstermekle beraber bundan sonraki çalışmalar için farklı varlık gruplarına özgü stratejiler geliştirilmesi önerilmektedir. 
Anahtar Kelimeler: Algoritmik trade, geçmişe dönük testler, getiri analizi, veri bilimi JEL Kodları: D53, F47, G11,

\section{REFERENCES}

Akkoyun, E. (2019, March). Growth Model For Abdominal Aortic Aneurysms Using. Orta Doğu Teknik Üniversitesi.

Aksoy, B. (2020). Finansal Piyasalarda Pay Senedi Manipülasyonunun Veri Madenciliği Yöntemleri İle Tahmini: Borsa İstanbul Örneği. Uluslararası İktisadi ve İdari İncelemeler Dergisi, 1-24.

al-Khwarazmi. (1989). al-Khwarazmi's Algebra. Al-Kitab al-Muhtasar fi Hesab al-Jabr wa'I-Mukabala. içinde Islamabad.

Bostancı, A. (2006). Riske Maruz Değer Hesaplama Yöntemlerinin Karşılaştırılması ve Geriye Dönük Test (Backtestıng) Uygulaması . Sosyal Bilimler, İşletme. Zonguldak Karaelmas Üniversitesi.

Chen, J. (2019, October 10). High-Frequency Trading (HFT) Definition. July 4, 2020 tarihinde investopedia: https://www.investopedia.com/terms/h/high-frequency-trading.asp adresinden alındı

Dictionary.Com. (1995). Dec 10, 2019 tarihinde Definition of Arbitrage on Dictionary.Com: https://www.dictionary.com/browse/arbitrage?s=t adresinden alındı

Dilaveroğlu, O. K. (2020). portföy getirilerinin riske uyarlanmiş performans değerlendirme ölçütleri ile incelenmesi: bist-30 endeksi üzerinde 2010-2019 yillarini kapsayan bir çalişma.

Enerji Piyasaları İşletme Anonim Şirketi Teşkilat Yapısı ve Çalışma Esasları Hakkında Yönetmelik. (2015). Resmi Gazete s. 29313.

EPIAŞ. (2020, June 30). GÖP: https://www.epias.com.tr/gun-oncesi-piyasasi/surecler/ adresinden alındı

EPIAŞ. (2020, June 06). EPIAŞ Şeffaflık Platformu: https://seffaflik.epias.com.tr/transparency/piyasalar/dengesizlik/dengesizlik-tutari.xhtml adresinden alındı

Erdoğan, N. (1995). Uluslararası İşletmelerde Mali Risk ve Yönetimi \& Çağdaş Finanman Teknikleri. Banka Uzmanları Derneği Yayınları.

Financial Post - Business. (2020, Feb 03). Feb 04, 2020 tarihinde China suffers most savage market rout in years, with over 3,000 stocks falling by daily limit within minutes: https://business.financialpost.com/investing/chinas-worst-rout-in-years-has-3257-stocks-falling-by-daily-limit adresinden alındı

Google Ngram Viewer. (tarih yok). Dec 18, 2019 tarihinde Google Books: https://books.google.com/ngrams/graph?content=algorithm adresinden alındı

Hayes, A. (2019, Jun 25). investopedia. Nov 2019 tarihinde Definition of Arbitrage Pricing Theory (APT): https://www.investopedia.com/terms/a/apt.asp adresinden alındı

IBM. (2011, 07 29). IBM. 07 04, 2020 tarihinde IBM 100 Deep Blue: https://www.ibm.com/ibm/history/ibm100/us/en/icons/deepblue/\#ai adresinden alındı

ICE Futures, U. I. (2015, June). U.S. Dollar Index ${ }^{\circledR}$ Contracts. https://www.theice.com/publicdocs/futures_us/ICE_Dollar_Index_FAQ.pdf adresinden alınd

Investing.Com. (2020). Feb 2020 tarihinde Shanghai Composite Index (SSEC): https://www.investing.com/indices/shanghai-composite adresinden alındı

KAP. (2019, Nov 13). Nov 2019 tarihinde KAP: https://www.kap.org.tr/tr/Bildirim/798746 adresinden alındı

KAP. (2019, Agu 08). KAP. Nov 2019 tarihinde KAP: https://www.kap.org.tr/tr/Bildirim/781239 adresinden alınd

Kızrak, M. A., \& Bolat, B. (2018). Derin Öğrenme ile Kalabalık Analizi Üzerine Detaylı Bir Araştırma. BiLişiM TEKNOLOJiLERi DERGisi, $263-286$.

Kurumsal Yatırımcı Dergisi. (2018, Nov Oct Dec 00). Güney Kore Fon Sektörü, s. 65.

Leviathan, Y., Matias, Y., \& Google Al Blog. (2018, May 08). Google Al Blog. July 04, 2020 tarihinde Google Duplex: An Al System for Accomplishing Real-World Tasks Over the Phone: https://ai.googleblog.com/2018/05/duplex-ai-system-for-natural-conversation.html adresinden alınd

Lexico on Oxford Dictionary. (2010). Dec 18, 2019 tarihinde Definition of Algorithm: https://www.lexico.com/en/definition/algorithm adresinden alındı

Lexico on Oxford Dictionary. (2010). 12 10, 2019 tarihinde https://www.lexico.com/en/definition/arbitrage adresinden alındı 
Marr, B. (2016, Apr). Forbes. Why Everyone Must Get Ready For The 4th Industrial Revolution. 2020 tarihinde https://www.forbes.com/sites/bernardmarr/2016/04/05/why-everyone-must-get-ready-for-4th-industrial-revolution/\#5ec184a83f90 adresinden alınd

McCorduck, P. (2004). Machines Who Think. A K Peters, Ltd.

Meeker, M. (2019). Optimizing the Sharpe Ratio with Portfolio Turnover, Reasoning and Methodology. Cambridge High School. Cambridge: A Quantopian Based Paper.

Memiş, C. (2018). backtesting the modified var and expected shortfall methods: for non-linear portfolios within basel accords. Engineering, Financial Engineering. İstanbul: Özyeğin University.

Nilsson, N. J. (2010). For Automated Trading. N. J. Nilsson içinde, The Quest For Artificial Intelligence (s. 626). Stanford University: Cambridge University Press.

Nilsson, N. J. (2010). Early AI Laboratories. The Quest For Artificial Intelligence. Stanford: Cambridge University Press.

Nilsson, N. J. (2010). The Quest For Artıfıcıal Intellıgence. Stanford University: Cambridge University Press.

ÖZMEN, C. (2019). Ethics Of Artificial Intelligence: Moral Responsibility Of Self-Driving Cars And Sex Robots. Orta Doğu Teknik Üniversitesi, Felsefe. Yüksek Öğrenim Kurumu.

ÖZMEN, M. C. (2019). Ethics Of Artificial Intelligence: Moral Responsibility Of Self-Driving Cars And Sex Robots. Sosyal Bilimler, Felsefe. Orta Doğu Teknik Üniversitesi.

Prado, M. L. (2016). Building diversified portfolios that outperform out-of-sample.: https://papers.ssrn.com/sol3/papers.cfm?abstract_id=2713516 adresinden alındı

QuantConnect.Com. (2020). QuantConnect. 2020 tarihinde Design and Trade Algoritmic Trading Strategies: Design and Trade Algoritmic Trading Strategies adresinden alındı

Quantiacs. (2020). July 05, 2020 tarihinde Learn about algorithmic trading: https://www.quantiacs.com/For-Quants/GetStarted.aspx adresinden alındı

Quantopian. (2020, 07 04). https://www.quantopian.com/faq. Quantopian: https://www.quantopian.com/faq adresinden alındı

QuantRocket. (2020, 2020). Quantrocket. Usage Guide: https://www.quantrocket.com/docs/?python\# adresinden alındı

Rodrigez, D. (2013). Backtrader. Jun 2, 2020 tarihinde Algotrading: https://www.backtrader.com/home/helloalgotrading/ adresinden alındı

Silicon Republic. (2014, October 10). Silicon Republic: https://www.siliconrepublic.com/play/15-billion-songs-have-been-identified-bymusic-recognition-service-shazam adresinden alındı

Toomer, G. (tarih yok). Al-Khwârizmi.

TSPB. (2018, Dec). Dec 18, 2019 tarihinde Sermaye Piyasası Zorlu Dönemi Başarıyla Atlattı: https://www.tspb.org.tr/tr/sermaye-piyasasizorlu-donemi-basariyla-atlatti/ adresinden alındı

Uyar, U. (2019). Makine Öğrenmesi İle Portföy Optimizasyonu: Ftse, Dax Ve Bist. 23. Finans Sempozyumu Marmara Üniversitesi İşletme Fakültesi (s. 161-175). Antalya: Marmara Üniversitesi İşletme Fakültesi.

Weiss, E. H. (1990). Visualizing a Procedure with Nassi-Schneiderman Charts. Journal of Technical Writing and Communication, $237-254$. 